\title{
THE IMMUNOPHYSIOLOGY OF TRICHINOSIS. ALTERATIONS IN THE BLOOD VOLUME AND THE THIOCYANATE SPACE IN RELATION TO THE DEVELOPMENT OF HUMORAL ANTIBODIES IN THE RABBIT ${ }^{1}$
}

\author{
By JERRY K. AIKAWA, GEORGE T. HARRELL, AND TREVA B. MILLER
}

\author{
(From the Department of Internal Medicine, Bowman Gray School of Medicine of Wake \\ Forest College, Winston-Salem, N. C.)
}

(Submitted for publication January 22, 1951; accepted, March 19, 1951)

The development of edema during the course of trichinosis has been observed in patients for many years. The accumulation of fluid, especially around the eyes, has been recognized as one of the most frequent clinical manifestations of the disease. The mechanism by which edema develops during the course of this and other acute infectious diseases has been obscure.

During the course of studies on patients with Rocky Mountain spotted fever, it was observed that alterations in the distribution of body fluids with the development of marked clinical edema reached a maximum just before clinical recovery begins (1). Observations on one patient who had been given hyperimmune rabbit antiserum for Rocky Mountain spotted fever showed that the alterations in the blood volume and thiocyanate space, which occurred during classic serum sickness following recovery from the initial disease, resembled strikingly those seen in the same patient during the acute stage of the rickettsial infection. These observations suggested the possibility that an immune reaction may be directly or indirectly responsible for the changes in the permeability of membranes which permit alteration in the fluid distribution in rickettsial spotted fever.

In subsequent studies on experimental serum sickness in rabbits, the alterations in the distribution of body fluids were found to resemble those encountered in patients with spotted fever (2). The time relationship between the maximal alterations and the appearance of circulating antibodies (precipitins) was interpreted as indicating that the increase in permeability of membranes was the result of an antigen-antibody reaction.

The present experiments were undertaken to determine the magnitude of changes in the blood

1 This study was aided by a grant from the John and Mary R. Markle Foundation. volume and thiocyanate space which occur during an interstitial infection such as trichinosis (as contrasted to the intracellular infection of spotted fever and the antigen-antibody reaction induced without infection of serum sickness) and to correlate the physiologic alterations with the development of humoral immunity.

\section{MATERIAL}

Domestic rabbits of mixed breeds, weighing 2 to 4 kilograms each, were placed in individual cages and fed a stock diet (Kasco Complete Rabbit Ration, Kasco Mills, Inc., Toledo, Ohio), supplemented once weekly by fresh green vegetables. Water was given without restriction.

The strain of Trichinella spiralis used was obtained from the National Institute of Health and was carried in white rats. The rats were killed six weeks after infection, and the skeletal muscle was pooled and chopped fine with scissors. A sample of the pooled meat was examined in a muscle press and found to contain approximately 120 encysted muscle trichinae per $\mathrm{Gm}$. It was fed to the rabbits within 24 hours.

\section{METHODS}

The details of the chemical techniques and experimental procedure for fluid volume determinations in rabbits have been previously published $(2,3)$.

Immunologic technique: The presence of circulating humoral antibody was detected by the ring precipitin test performed in $2 \times 20 \mathrm{~mm}$. tubes. Undiluted rabbit serum (antibody) was overlayed with a 1:200 dilution in saline of powdered muscle trichinae (antigen) prepared by Bozicevich's method (4).

Plan of the experiment: Determinations of the blood volume, thiocyanate space and precipitin tests were done simultaneously every second or third day. A minimum of eight determinations was done on each animal. The two groups were treated in exactly the same fashion except for the feeding of trichinous rat meat. Uninfected meat was not fed to the rabbits in group 1, since previous experiments had failed to demonstrate that the feeding of uninfected rat meat can produce sensitization to foreign proteins (5). The six control animals (group 1) were followed at least 30 days. 


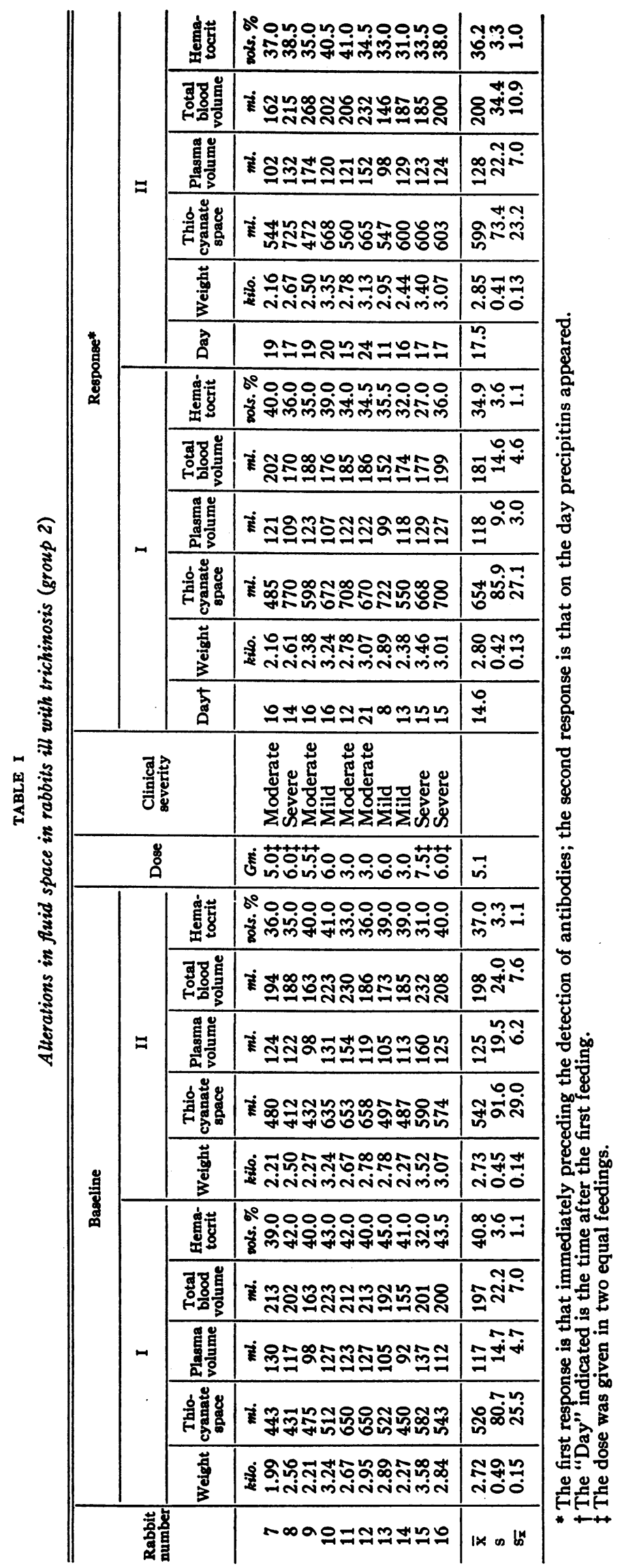


After a baseline for the individual animal had been established, the 10 rabbits in group 2 were each forced to eat 3 to $7.5 \mathrm{Gm}$. of the trichinous rat meat (Table I). Five of the animals were fed half of the infecting dose on each of two successive days. All of the animals in group 2 were followed at least 30 days following the feeding of trichinous meat, unless accidental death from hemopericardium supervened.

Statistical analysis: The details of the statistical methods have been previously described (2). The total blood volume, plasma volume, hematocrit and thiocyanate space were recorded in absolute values (Table I). The mean of two successive baseline determinations on each animal was compared with the mean of two successive determinations made just before the precipitin test became positive and on the day precipitins appeared. In this fashion, each animal in the experimental group (group 2) served as its own control.

The data as obtained in absolute values were then calculated in terms of milliliters per kilogram of body weight on the day the measurement was made.

The data were further analyzed comparing the first baseline determination with the determination made just prior to the appearance of precipitins, and comparing the first baseline determination with the determination made on the day precipitins were first detected. These response values were also analyzed in terms of absolute values and of milliliters per kilogram of initial body weight.

\section{RESULTS}

Control on methods: The data in the uninfected animals (group 1) are in accord with those previously published and are not presented in detail since they are not utilized in the statistical calculations in the infected animals (group 2) $(2,3)$.

General observations: None of the animals in group 1 showed any symptoms or signs suggesting trichinosis. No reaction was noted in any rabbit to the repeated intravenous injection of dye and no evidence of sensitization was detected by skin or precipitin test.

Clinical evidence of infection appeared in seven of 10 animals in group 2 within the first 10 days following the feeding of trichinous rat meat. In five of these animals the skin became soggy, hot, and moist between the fifth and ninth days and remained so for a maximum of five days. This edema was most striking in the skin over the abdomen but was not evident unless the fur had been clipped. In the other two animals the initial sign of infection was the appearance of a thin, hot, dry skin between the eighth and 10th days. In these seven animals the clinical signs reached a maximum and largely subsided before the precipitin test became positive.
Three animals developed clinical signs after the 10th day of feeding. In one the skin became hot and dry on the 15th day-the time at which a positive precipitin test was first detected. Another rabbit developed edema after the appearance of a positive precipitin test.

In general the clinical severity of early trichinosis appeared to be directly related to the number of muscle trichinae ingested (Table I). The rabbit fed the largest amount of trichinous meat (7.5 $\mathrm{Gm}$.) developed the severest clinical edema. The three animals in which clinical signs appeared late in the course of observation had been fed the minimal dose of rat meat $(3 \mathrm{Gm}$.). In general the changes in the distribution of body fluid measured paralleled the clinical severity of the disease process.

At the conclusion of the experiment a sample of meat from each rabbit was examined by either the muscle press or digestion technique; all the animals in group 2 were infected.

Four of the animals appeared jaundiced; one on the fifth, two on the 10th, and one on the 11th day after feeding.

Immunologic findings: In all 10 animals in group 2 precipitin tests to trichinella antigen became positive and remained so during the period of observation; the distribution was as follows :

\begin{tabular}{l|r|r|r|r|r|r|r}
\hline \hline & \multicolumn{6}{|c}{ Onset of positive precipitin tests } \\
\hline $\begin{array}{l}\text { Days after feeding } \\
\text { Number of rabbits }\end{array}$ & 11 & 15 & 16 & 17 & 19 & 20 & 24 \\
1 & 1 & 1 & 3 & 2 & 1 & 1 \\
\hline
\end{tabular}

Thiocyanate space: Increases in the thiocyanate space developed at some time during the period of observation in all of the animals in group 2 (Table I). In all except two the increase in thiocyanate space had begun to subside before the precipitin test became positive. In six animals the thiocyanate space increased a second time following the development of a positive precipitin test (Figure 1).

The alteration in thiocyanate space did not parallel the changes in body weight. At some time during the period of observation, in all animals, the body weight did not change appreciably or increase as the thiocyanate space increased. As the thiocyanate space subsequently decreased, this alteration was again out of proportion to the change 
TRICHINOSIS IN RABBIT

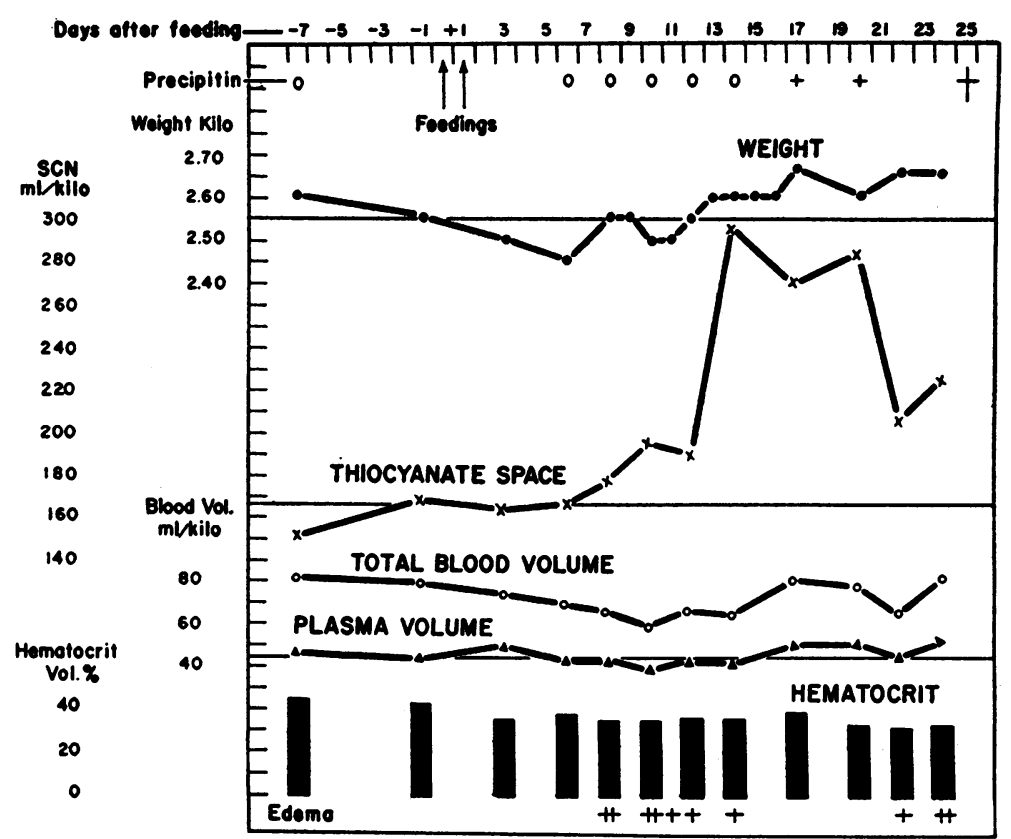

Fig. 1. Fluid Changes in a Rabbit Fed 6 Gm. of Trichinous Rat Meat

Note the increase in thiocyanate space on the 14th day after the first feeding, without a concomitant relative increase in body weight. The blood volume was decreased slightly during the 10th to 14th days. This rabbit was severely ill; it developed clinical jaundice on the 10th day. The physiological changes were reversing when the precipitin became positive on the 17th day.

in body weight and could not be accounted for on this basis alone.

When the mean of the two baseline determinations is compared statistically with the mean of the two response values recorded in the table, the value of $\bar{d}$ is found to be $+90.6 ; s \bar{d} 29.1 ; t 3.11$, giving a value for $P$ between 0.01 and 0.02 . When the analysis is performed utilizing the first baseline and the data obtained just before the appearance of the positive precipitin test, the data are highly significant whether analyzed in terms of absolute values or of milliliters per kilogram of weight on that day. The corresponding figures are, respectively: $\overline{\mathrm{d}}+128.5, \mathrm{~s} \overline{\mathrm{d}} 29.4, \mathrm{t} 4.37, \mathrm{P}$ less than 0.01 ; and $\bar{d}+39.8, s \bar{d} 11.8, t 3.37, P$ less than 0.01 . When the first baseline value is compared with the first response in terms of milliliters per kilogram of initial (baseline) body weight, the figures are also highly significant with $t 4.25$ and $P$ less than 0.01 .

Blood volume: Alterations in the plasma volume were not significant by any means of calculation.
When alterations in the total blood volume were calculated in terms of milliliters per kilogram of body weight on the day of the first response and were compared with the first baseline determination, the value of $t$ was 3.25 with $\mathrm{P} 0.01$. No other values were significant.

On the whole the minor decreases in blood volume tended to precede the development of a positive precipitin test and paralleled the clinical severity of the disease.

Hematocrit: A steady decrease in hematocrit was observed early in the experiment and tended to disappear with recovery. The values were statistically significant with $P$ less than 0.01 when the first baseline value was compared with either the first or second response value. The $t$ values were respectively $6.13,3.55$.

Weight: The changes in weight were not considered statistically significant since the value for $P$ was always greater than 0.05 . 


\section{DISCUSSION}

The experiments indicate that rabbits experimentally infected with trichinosis exhibit alterations in the fluid space which is available for the diffusion of thiocyanate ion. The maximal alterations occur just before the appearance of circulating humoral antibodies (precipitins) in the serum. This finding correlates well with the previous clinical observation that the symptoms of serum sickness begin to disappear with the appearance of precipitins (6).

In trichinosis, however, the immunologic problem is more complicated than in serum sickness. Serum disease is usually produced by a single injection, containing all antigens, and the response is dramatic. In trichinosis, on the other hand, multiple antigens are being continuously introduced over a period of weeks. The two increases in the thiocyanate space detected in some animals may be the result of antibody responses to two distinct antigens. Differences have been suspected in the antigens contained in the bodies of adult and larval forms of trichinae (7); it is possible that the antigenic components of immature, unencysted trichinella larvae may also be slightly different from the mature encysted forms. Previous studies have demonstrated that peptic and tryptic digestion destroys the protein fraction of the larvae, but does not destroy the antigenicity of some remaining component which presumably is a carbohydrate (8). The adult female attaches to the intestinal mucosa and secretes an antigen which probably is of little significance in the acute disease; the antibody, however, is concerned with the prevention of re-infection. Because of the relatively small number of adults in relation to the huge number of larvae, it is likely that the larval antigens are of greater concern in acute trichinosis. In any event, the physiologic alterations caused by different antigenic substances appear to vary quantitatively but not qualitatively.

Permeability of capillary membranes: Observations on human beings with rickettsial spotted fever and on rabbits with experimental serum sickness have been interpreted as indicating that alterations in the permeability of the vascular tree occurred $(1,2)$. The degree of alteration varied with the clinical severity of the disease process. In mild instances water and ions which normally traverse the capillary membrane would leave the blood more readily. Larger molecules such as protein would be lost in more severe instances and red blood cells would pass out of the vascular tree only in the most severe reactions.

During the stage of edema of the muscles in severe clinical trichinosis the accumulation of fluid is associated with oliguria which lasts from one to two weeks and is followed by diuresis. This sequence of events is also observed during pneumonia and serum disease and is accompanied by retention of water, sodium and chloride $(9,10)$. These clinical findings can be explained by an increase in capillary permeability; water and ions lost from the vascular tree must be retained in the tissues.

When serial determinations of the blood proteins have been made in clinically severe instances of the disease hypoproteinemia has been found (11). The minimal decreases in blood volume detected by the dye method indicate that in the experiments reported the alteration in capillary permeability to molecules larger than those of salt and water was equivocal. Rough determinations of the serum proteins by a microtechnique showed only slight alterations in these rabbits. Protein levels in the plasma and interstitial fluid of patients with the disease should be followed serially, especially during the stage of edema, to determine whether proteins leak into interstitial spaces. Information on the rate of protein excretion is also needed to estimate the degree of tissue destruction and of protein loss.

The drop in the hematocrit without evidence of intravascular hydration indicates that red blood cells were lost from the vascular compartment. The drop was apparently not due to the repeated cardiac punctures since the hematocrit remained constant in group 1 and other experiments have shown that the amount of blood withdrawn is inadequate to produce this change $(2,3)$. No petechiae were noted and it is unlikely that the degree of hemorrhage which accompanies the breaking out of larvae from capillaries into interstitial muscle spaces is adequate to explain the drop in hematocrit. It is known that the trauma which occurs during penetration of gravid trichinae into the mucosa of the small intestine results in microscopic ulceration and bleeding. No evidence has been presented, however, that intestinal trichinae ingest 
red blood cells in the manner that the hookworm does. The icteric tint of the serum and clinical jaundice observed in four rabbits during the period when the blood volume was reduced suggest that some red cells may have been hemolyzed. Intravascular hemolysis results from antigen-antibody reactions which directly involve red blood cells, but no such process is recognized in trichinosis.

Cellular permeability: It is not known whether the thiocyanate ion normally diffuses into cells or not. The fact that some rabbits showed an increase in thiocyanate space without a comparable increase in body weight would favor the thesis that some re-distribution of fluid had occurred, whether or not additional fluid ingested in food or water was retained. It would appear that the increase in thiocyanate space is not due entirely to an increase in fluid between cells but is indicative of increased hydration within cells. If this be true, the reversible psychic disturbances seen during the stage of edema in acute clinical trichinosis may be due to excessive hydration of nerve cells.

The appearance of positive skin tests in patients with trichinosis has been interpreted as indicating the development in the disease of cellular immunity and hypersensitivity which often is of marked degree. It is likely that the cellular component of immunity of trichinosis is greater than the humoral component. If the appearance of circulating antibodies is indicative of prior neutralization of antigens attached to cells as well as circulating in the blood, the maximum physiologic alterations resulting from their interaction would precede the detection of humoral immunity. Such was the case. Apparently an antigen-antibody reaction occurred either on the cell surface (as probably occurs in serum sickness) or within the cell (as in rickettsial disease); the cell was injured and the permeability of the cell membrane to the thiocyanate ion was increased.

Mechanisms: It has been postulated that histamine or some similar substance is released during the interaction of antigens with antibodies. The liberation at the cell or capillary wall of some substance which would increase the permeability of the cell membranes would enhance the transmission of molecules including the thiocyanate ion across the surface. It is not known whether the antigens in trichinosis attach to the vascular wall or to tissue cells outside the vascular system as well. The anatomic location of trichinella larvae in the interstitial spaces would favor the latter hypothesis.

In the microscopic examination of human muscle, when no trichinae are found it is sometimes difficult to differentiate trichinosis from periarteritis nodosa, and a possible relationship between the two diseases has been suggested (12). Lesions resembling periarteritis have been found in rabbits sensitized to horse serum (13). The above observations add further support to the view that the symptoms and signs of clinical trichinosis may be at least partially due to sensitization of the host to antigenic substances contained in or secreted by trichinae.

The present study indicates that the physiologic alterations which may result from antigen-antibody reactions occurring during an interstitial infection are more profound than has been appreciated in the past. The principles of supportive therapy previously described in Rocky Mountain spotted fever which emphasize alterations in permeability of membranes may be usefully applied to the treatment of patients with trichinosis (14). The possibility that the administration of antihistaminic drugs may decrease the physiologic alterations resulting from the immune or allergic reactions should be considered.

\section{SUM MARY}

1. Trichinosis, an extracellular infection, is accompanied in rabbits by clinical edema and an increase in the fluid space available for dilution of thiocyanate ions.

2. The changes were interpreted as indicating an increase in permeability of the vascular tree, probably in the capillary wall, so that crystalloids were lost from the circulation into the interstitial spaces.

3. Occasional discrepancies observed between the increase in thiocyanate space and the changes in weight suggest that cellular permeability is probably also increased to water and ions.

4. A significant drop in hematocrit occurred; in occasional animals it was accompanied by clinical jaundice and the appearance of icterus in the serum.

5. The physiologic changes were maximal just before humoral antibodies( precipitins) appeared, suggesting that the alterations may have been due 
directly or indirectly to an antigen-antibody reaction.

6. The clinical symptoms and signs of trichinosis are not solely due to mechanical blockage of capillaries or arterioles by migrating larvae, but are more likely the result of re-distribution of fluid in the extravascular spaces.

\section{REFERENCES}

1. Harrell, G. T., and Aikawa, J. K., The pathogenesis of circulatory failure in Rocky Mountain spotted fever. Alterations in the blood volume and the thiocyanate space at various stages of the disease. Arch. Int. Med., 1949, 83, 331.

2. Aikawa, J. K., and Harrell, G. T., The immunophysiology of serum sickness. Alterations in the blood volume and thiocyanate space in relation to the development of humoral antibodies in the rabbit. J. Clin. Invest., 1951, 30, 360.

3. Aikawa, J. K., Fluid volumes and electrolyte concentrations in normal rabbits. Am. J. Physiol., 1950, $162,695$.

4. Bozicevich, J., Studies on trichinosis, XII. The preparation and use of an improved trichina antigen. Pub. Health Rep., 1938, 53, 2130.

5. Avera, J. W., Yow, E. M., Harrell, G. T., and Fowler, E. B., An attempt by feeding to induce in animals reactivity to Trichinella spiralis in the absence of infection. Am. J. Trop. Med., 1946, 26, 125.
6. Longcope, W. T., and Rackemann, F. M., The relation of circulating antibodies to serum disease. J. Exper. 'Med., 1918, 27, 341.

7. Oliver-González, J., The dual antibody basis of acquired immunity in trichinosis. J. Infect. Dis., 1941, 69, 254.

8. Aikawa, J. K., Harrell, G. T., and Helsabeck, N. J., The effect of peptic and tryptic digestion on the antigenicity of Trichinella spiralis. J. Clin. Invest., 1947, 26, 73.

9. Rutstein, D. D., Thomson, K. J., Tolmach, D. M., Walker, W. H., and Floody, R. J., Plasma volume and "extravascular thiocyanate space" in pneumococcus pneumonia. J. Clin. Invest., 1945, $24,11$.

10. Rackeriann, F. M., Longcope, W. T., and Peters, J. $P$. ., The excretion of chlorids and water and the renal function in serum disease. Arch. Int. Med., 1916, 18, 496.

11. Hanes, F. M., Trichinosis complicated by hypoproteinemia. Internat. Clin., 1936, 4, 67.

12. Reimann, H. A., Price, A. H., and Herbut, P. A., Trichinosis and periarteritis nodosa; differential diagnosis; possible relationship. J.A.M.A., 1943, $122,274$.

13. Rich, A. R., and Gregory, J. E., The experimental demonstration that periarteritis nodosa is a manifestation of hypersensitivity. Bull. Johns Hopkins Hosp., 1943, 72, 65.

14. Harrell, G. T., Rocky Mountain spotted fever. Medicine, 1949, 28, 333. 